\title{
A Dirac System with Transmission Condition and Eigenparameter in Boundary Condition
}

\author{
Abdullah Kablan and Tülay Özden \\ Department of Mathematics, Faculty of Arts and Sciences, Gaziantep University, 27310 Gaziantep, Turkey \\ Correspondence should be addressed to Abdullah Kablan; kablan@gantep.edu.tr
}

Received 5 May 2013; Accepted 11 July 2013

Academic Editor: Ravshan Ashurov

Copyright (c) 2013 A. Kablan and T. Özden. This is an open access article distributed under the Creative Commons Attribution License, which permits unrestricted use, distribution, and reproduction in any medium, provided the original work is properly cited.

This paper deals with a Dirac system with transmission condition and eigenparameter in boundary condition. We give an operatortheoretic formulation of the problem then investigate the existence of the solution. Some spectral properties of the problem are studied.

\section{Introduction}

After Walter [1] had given an operator-theoretic formulation of eigenvalue problems with eigenvalue parameter in the boundary conditions, Fulton $[2,3]$ has carried over the methods of Titchmarsh [4, chapter 1$]$ to this problem. Then, a large amount of the mathematical literature was devoted to these subjects during the last twenty years. We will mention some of the papers published at least twenty years ago, but of course there are many other interesting and important papers published more recently, which are not referred to here. The existence of solution and some spectral properties of Sturm-Liouville problem with eigenparameter-dependent boundary conditions and also with transmission conditions at one or more inner points of considered finite interval has been studied by Mukhtarov and Tunç [5]; see also [6, 7]. A Dirac system when the eigenparameter appears in boundary conditions has been studied by Kerimov [8]. In [9], an inverse problem for the Dirac system with eigenvaluedependent boundary conditions and transmission condition is investigated.

The aim of the present paper is to study a Dirac system with transmission condition and eigenparameter in boundary condition. For this, we follow the method in [5]. We consider the Dirac system

$$
\ell(u)=A u^{\prime}(x)-P(x) u(x)=\lambda u(x)
$$

where

$$
\begin{gathered}
A=\left(\begin{array}{cc}
0 & 1 \\
-1 & 0
\end{array}\right), \\
P(x)=\left(\begin{array}{cc}
p_{1}(x) & 0 \\
0 & p_{2}(x)
\end{array}\right), \\
u(x)=\left(\begin{array}{l}
u_{1}(x) \\
u_{2}(x)
\end{array}\right),
\end{gathered}
$$

or

$$
\begin{aligned}
& u_{2}^{\prime}(x)-p_{1}(x) u_{1}(x)=\lambda u_{1}(x), \\
& u_{1}^{\prime}(x)+p_{2}(x) u_{2}(x)=-\lambda u_{2}(x), \quad x \in[a, c) \cup(c, b],
\end{aligned}
$$

with boundary conditions

$$
\begin{gathered}
\sin \alpha u_{1}(a)-\cos \alpha u_{2}(a)=0, \\
b_{1} u_{1}(b)-a_{1} u_{2}(b)+\lambda\left(\sin \beta u_{1}(b)-\cos \beta u_{2}(b)\right)=0,
\end{gathered}
$$

and transmission conditions at the inner point $x=c$

$$
\begin{gathered}
u_{1}(c-0)=\gamma u_{1}(c+0), \\
u_{2}(c-0)=\gamma^{-1} u_{2}(c+0) .
\end{gathered}
$$

Here and later on, $\lambda$ is a complex eigenvalue parameter; the functions $p_{i}(x)(i=1,2)$ are continuous on $[a, c) \cup(c, b]$ which have finite limits $p_{i}( \pm c)=\lim _{x \rightarrow \pm c} p_{i}(x)(i=1,2), a_{1}, b_{1}, \gamma$ are real numbers and $\alpha, \beta \in[0, \pi)$. 


\section{Operator Formulation of the Problem}

For convenience, we will assume that $\left|a_{1}\right|+\left|b_{1}\right| \neq 0, \gamma \neq 0$. To formulate a theoretic approach to problem (1)-(6), we define the Hilbert space $\mathbb{U}=L_{2}[a, c) \cup L_{2}(c, b] \oplus \mathbb{C}_{\sigma}$ with an inner product

$$
\langle U, V\rangle_{\mathbb{H}}=\int_{a}^{c} u^{T}(x) \bar{v}(x) d x+\int_{c}^{b} u^{T}(x) \bar{v}(x) d x+\frac{1}{\sigma} \widetilde{u} \bar{v},
$$

where $T$ stands for the transpose and

$$
\begin{array}{cc}
U=\left(\begin{array}{c}
u(x) \\
\tilde{u}
\end{array}\right), & V=\left(\begin{array}{c}
v(x) \\
\widetilde{v}
\end{array}\right) \in \mathbb{H}, \\
u(x)=\left(\begin{array}{c}
u_{1}(x) \\
u_{2}(x)
\end{array}\right), & v(x)=\left(\begin{array}{c}
v_{1}(x) \\
v_{2}(x)
\end{array}\right) \in H,
\end{array}
$$

$u_{i}(x), v_{i}(x) \in L_{2}[a, c) \cup L_{2}(c, b],(i=1,2), \tilde{u}, \tilde{v} \in \mathbb{C}$. The constant $\sigma$ is defined by

$$
\sigma:=\operatorname{det}\left(\begin{array}{cc}
b_{1} & a_{1} \\
\sin \beta & \cos \beta
\end{array}\right)>0
$$

Let $\operatorname{dom}(\mathbf{A}) \subseteq \mathbb{W}$ be set of all $U=\left(\begin{array}{c}u(x) \\ \widehat{u}\end{array}\right) \in \mathbb{M}$, such that $u_{1}(x), u_{2}(x)$ are absolutely continuous on $[a, c) \cup(c, b], \widehat{u}=$ $\sin \beta u_{1}(b)-\cos \beta u_{2}(b)$ and $\ell(u) \in \mathbb{H}, \sin \alpha u_{1}(a)-\cos \alpha u_{2}(a)=$ $0, u_{1}( \pm c), u_{2}( \pm c)$ have finite limits, $\tilde{u}=b_{1} u_{1}(b)-a_{1} u_{2}(b)$. Now define the operator $\mathbf{A}: \operatorname{dom}(\mathbf{A}) \rightarrow \mathbb{W}$ by

$$
\mathbf{A}\left(\begin{array}{c}
u(x) \\
\widehat{u}
\end{array}\right)=\left(\begin{array}{c}
\ell(u) \\
-\widetilde{u}
\end{array}\right)
$$

Hence, we can rewrite the problem (1)-(6) in the operator form as

$$
\mathbf{A} U=\lambda U
$$

Obviously, the operator A and the Dirac system (1)-(6) have the same eigenvalues. Also the eigenvectors of (1)-(6) coincide with the first two components of the corresponding eigenelement of the operator $\mathbf{A}$.

Lemma 1. The $\operatorname{dom}(\mathbf{A})$ is dense in $\mathbb{H}$.

Proof. It is easily seen that there is no nonzero vector $F=$ $(f(x), \widehat{f}) \in \mathbb{W}$ such that for every $U=(u(x), \widehat{u}) \in \operatorname{dom}(\mathbf{A})$, $\langle F, U\rangle_{\mathbb{⿴}}=0$. This implies $\operatorname{dom}(\mathbf{A})^{\perp}=\{\Theta\}$, where $\Theta=$ $(0,0,0)$. Therefore, $\operatorname{dom}(\mathbf{A})$ is dense in $\mathbb{H}$.

Theorem 2. The operator $\mathbf{A}$ is symmetric.

Proof. For each $U, V \in \operatorname{dom}(\mathbf{A})$ from the inner product (7) and the integration by parts, we have

$$
\langle\mathbf{A} U, V\rangle_{\square}=\int_{a}^{c}\left(u_{2}^{\prime}-p_{1} u_{1}\right) \bar{v}_{1} d x-\int_{a}^{c}\left(u_{1}^{\prime}+p_{2} u_{2}\right) \bar{v}_{2} d x
$$

$$
\begin{aligned}
& +\int_{c}^{b}\left(u_{2}^{\prime}-p_{1} u_{1}\right) \bar{v}_{1} d x \\
& -\int_{c}^{b}\left(u_{1}^{\prime}+p_{2} u_{2}\right) \bar{v}_{2} d x-\frac{1}{\sigma} \tilde{u} \bar{v} \\
& =\left[u_{2} \bar{v}_{1}-u_{1} \bar{v}_{2}\right]_{a}^{c-0}+\left[u_{2} \bar{v}_{1}-u_{1} \bar{v}_{2}\right]_{c+0}^{b} \\
& -\int_{a}^{c} u_{2} \bar{v}_{1}^{\prime} d x-\int_{a}^{c} p_{1} u_{1} \bar{v}_{1} d x+\int_{a}^{c} u_{1} \bar{v}_{2}^{\prime} d x \\
& -\int_{a}^{c} p_{2} u_{2} \bar{v}_{2} d x-\int_{c}^{b} u_{2} \bar{v}_{1}^{\prime} d x-\int_{c}^{b} p_{1} u_{1} \bar{v}_{1} d x \\
& +\int_{c}^{b} u_{1} \bar{v}_{2}^{\prime} d x-\int_{c}^{b} p_{2} u_{2} \bar{v}_{2} d x-\frac{1}{\sigma} \widetilde{u} \bar{v} \\
& =\left[u_{2}(c-0) \bar{v}_{1}(c-0)-u_{1}(c-0) \bar{v}_{2}(c-0)\right] \\
& -\left[u_{2}(a) \bar{v}_{1}(a)-u_{1}(a) \bar{v}_{2}(a)\right] \\
& +\left[u_{2}(b) \bar{v}_{1}(b)-u_{1}(b) \bar{v}_{2}(b)\right] \\
& -\left[u_{2}(c+0) \bar{v}_{1}(c+0)-u_{1}(c+0) \bar{v}_{2}(c+0)\right] \\
& -\int_{a}^{c} u_{2}\left(\bar{v}_{1}^{\prime}+p_{2} \bar{v}_{2}\right) d x+\int_{a}^{c} u_{1}\left(\bar{v}_{2}^{\prime}-p_{1} \bar{v}_{1}\right) d x \\
& -\int_{c}^{b} u_{2}\left(\bar{v}_{1}^{\prime}+p_{2} \bar{v}_{2}\right) d x+\int_{c}^{b} u_{1}\left(\bar{v}_{2}^{\prime}-p_{1} \bar{v}_{1}\right) d x \\
& -\frac{1}{\sigma}\left(b_{1} u_{1}(b)-a_{1} u_{2}(b)\right) \\
& \times\left(\sin \beta \bar{v}_{1}(b)-\cos \beta \bar{v}_{2}(b)\right) .
\end{aligned}
$$

Since $U$ and $V$ satisfy the same boundary condition (4) at $x=$ $a$,

$$
u_{2}(a) \bar{v}_{1}(a)=u_{1}(a) \bar{v}_{2}(a) .
$$

From transmission condition (6), it follows that

$$
\begin{aligned}
& u_{2}(c-0) \bar{v}_{1}(c-0)=u_{2}(c+0) \bar{v}_{1}(c+0), \\
& u_{1}(c-0) \bar{v}_{2}(c-0)=u_{1}(c+0) \bar{v}_{2}(c+0) .
\end{aligned}
$$

Furthermore,

$$
\begin{aligned}
& {\left[u_{2}(b) \bar{v}_{1}(b)-u_{1}(b) \bar{v}_{2}(b)\right]-\frac{1}{\sigma}\left(b_{1} u_{1}(b)-a_{1} u_{2}(b)\right)} \\
& \times\left(\sin \beta \bar{v}_{1}(b)-\cos \beta \bar{v}_{2}(b)\right) \\
& \quad=-\frac{1}{\sigma}\left(\sin \beta u_{1}(b)-\cos \beta u_{2}(b)\right)\left(b_{1} \bar{v}_{1}(b)-a_{1} \bar{v}_{2}(b)\right) \\
& \quad=-\frac{1}{\sigma} \widehat{u} \overline{\widetilde{v}} .
\end{aligned}
$$

Now substituting (13), (14), and (15) in (12), we obtain

$$
\langle\mathbf{A} U, V\rangle_{\mathbb{Q}}=\langle U, \mathbf{A} V\rangle_{\mathbb{W}} \cdot
$$


Since the operator $\mathbf{A}$ is symmetric, the following orthogonality relation is valid.

Corollary 3. All the eigenvalues of the system (1)-(6) are real and to every eigenvalue $\lambda_{n}$, there corresponds a vector-valued eigenfunction $u_{n}^{T}\left(x, \lambda_{n}\right)=\left(u_{1 n}\left(x, \lambda_{n}\right), u_{2 n}\left(x, \lambda_{n}\right)\right)$. Moreover, vector-valued eigenfunctions belonging to different eigenvalues are orthogonal in the sense of

$$
\left\langle u_{n}, u_{m}\right\rangle_{\mathbb{Q}}=\int_{a}^{c} u_{n}^{T} \bar{u}_{m} d x+\int_{c}^{b} u_{n}^{T} \bar{u}_{m} d x-\frac{1}{\sigma} \widetilde{u}_{n} \overline{\widehat{u}}_{m}=0 .
$$

Remark 4. The vector-valued eigenfunctions stated in Corollary 3 are not orthogonal in the usual sense in the Hilbert space $L_{2}[a, b]$.

\section{Existence of Solutions}

In this section, we study the existence of the solution of the Dirac system (1) with boundary conditions (4) and transmission condition (6).

Theorem 5. The Dirac system (1) has a solution $\Phi(x, \lambda)$ on $[a, b]$ satisfying boundary condition (4) and transmission condition (6). For each $x, \Phi(x, \lambda)$ is a vector-valued entire function of $\lambda$.

Proof. From the classical theory of differential equations (see [10]), since the Dirac system

$$
A u^{\prime}(x)-P(x) u(x)=\lambda u(x), \quad x \in[a, c)
$$

with the initial conditions

$$
u_{1}(a)=\cos \alpha, \quad u_{2}(a)=\sin \alpha
$$

is continuous on the interval $[a, c)$, this system has a unique solution $\Phi_{1}(x, \lambda)=\left(\Phi_{11}(x, \lambda), \Phi_{21}(x, \lambda)\right)^{T}$ which is an entire function of $\lambda$ on $[a, c)$.

Now consider the Dirac system of differential equations

$$
\begin{gathered}
u_{2}^{\prime}(x)-p_{1}(x) u_{1}(x)=\lambda u_{1}(x), \\
u_{1}^{\prime}(x)+p_{2}(x) u_{2}(x)=-\lambda u_{2}(x), \quad x \in(c, b],
\end{gathered}
$$

and nonstandard initial conditions contain eigenparameter

$$
\begin{gathered}
u_{1}(c+0)=\gamma^{-1} \Phi_{11}(c-0, \lambda), \\
u_{2}(c+0)=\gamma \Phi_{21}(c-0, \lambda) .
\end{gathered}
$$

Let us denote solutions of (20) by $u_{0}(x, \lambda)=\left(u_{10}(x, \lambda)\right.$, $\left.u_{20}(x, \lambda)\right)^{T}$ in the case $p_{1}(x)=p_{2}(x) \equiv 0$. It is clear that the vector-valued function $u_{0}(x, \lambda)$ is written as

$$
\begin{gathered}
u_{10}(x, \lambda)=c_{1} \cos \lambda x+c_{2} \sin \lambda x, \\
u_{20}(x, \lambda)=-c_{1} \sin \lambda x+c_{2} \cos \lambda x .
\end{gathered}
$$

From the initial conditions (21), we obtain constants $c_{1}$ and $c_{2}$. Then, inserting these values into (22) and using some basic trigonometric identities, we arrive at

$$
u_{0}(x, \lambda)=\left(\begin{array}{l}
u_{10}(x, \lambda) \\
u_{20}(x, \lambda)
\end{array}\right)=\left(\begin{array}{l}
\gamma^{-1} \Phi_{11}(c-0, \lambda) \cos \lambda(x-(c+0))+\gamma \Phi_{21}(c-0, \lambda) \sin \lambda(x-(c+0)) \\
\gamma_{1}^{-1} \Phi_{11}(c-0, \lambda) \sin \lambda(x-(c+0))+\gamma \Phi_{21}(c-0, \lambda) \cos \lambda(x-(c+0))
\end{array}\right) .
$$

By applying the method of variation of the constants as in [11, page 243], we find the following system of integral equations:

$$
u(x, \lambda)=\left(\begin{array}{l}
u_{1}(x, \lambda) \\
u_{2}(x, \lambda)
\end{array}\right)=\left(\begin{array}{l}
u_{10}(x, \lambda)+\int_{c}^{x}\left\{p_{1}(s) u_{1}(x, \lambda) \sin \lambda(s-x)-p_{2}(s) u_{2}(x, \lambda) \cos \lambda(s-x)\right\} d s \\
u_{20}(x, \lambda)+\int_{c}^{x}\left\{p_{1}(s) u_{1}(x, \lambda) \cos \lambda(s-x)+p_{2}(s) u_{2}(x, \lambda) \sin \lambda(s-x)\right\} d s
\end{array}\right) .
$$

In what follows, we use the method of successive approximations, which is helpful in constructing a solution of the integral equation system (24). This method requires a sequence of functions $\left\{u_{n}(x, \lambda)\right\}$ for $n=1,2, \ldots$ defined as

$$
u_{n}(x, \lambda)=\left(\begin{array}{l}
u_{1 n}(x, \lambda) \\
u_{2 n}(x, \lambda)
\end{array}\right)=\left(\begin{array}{l}
u_{10}(x, \lambda)+\int_{c}^{x}\left\{p_{1}(s) u_{1 n-1} \sin \lambda(s-x)-p_{2}(s) u_{2 n-1} \cos \lambda(s-x)\right\} d s \\
u_{20}(x, \lambda)+\int_{c}^{x}\left\{p_{1}(s) u_{1 n-1} \cos \lambda(s-x)+p_{2}(s) u_{2 n-1} \sin \lambda(s-x)\right\} d s
\end{array}\right)
$$

where $u_{10}(x, \lambda)$ and $u_{20}(x, \lambda)$ are defined in (23). It is obvious that each of $u_{n}(x, \lambda)$ is an entire function of $\lambda$ for every $x \in$ $(c, b]$.
Set

$$
z_{n}(x, \lambda)=u_{n}(x, \lambda)-u_{n-1}(x, \lambda),
$$


where $z_{n}^{T}(x, \lambda)=\left(z_{1 n}(x, \lambda), z_{2 n}(x, \lambda)\right)$, and let $M_{1}=$ $\max _{x \in(c, b]}\left|p_{1}(x)\right|, M_{2}=\max _{x \in(c, b]}\left|p_{2}(x)\right|, M=\max \left(M_{1}, M_{2}\right)$, $N_{1}(\lambda)=\max _{x \in(c, b]}\left|u_{10}(x, \lambda)\right|, N_{2}(\lambda)=\max _{x \in(c, b]}\left|u_{20}(x, \lambda)\right|$. Then,

$$
\begin{gathered}
\left\|z_{1}(x, \lambda)\right\| \leq \int_{c}^{x} \mid p_{1}(s) u_{10} \sin \lambda(s-x) \\
\quad-p_{2}(s) u_{20} \cos \lambda(s-x) \mid d s \\
+\int_{c}^{x} \mid p_{1}(s) u_{10} \cos \lambda(s-x) \\
\quad+p_{2}(s) u_{20} \sin \lambda(s-x) \mid d s \\
\leq 2 M\left(N_{1}(\lambda)+N_{2}(\lambda)\right)(x-c),
\end{gathered}
$$

where the norm $\|\cdot\|$ can be any convenient norm in $\mathbb{H}$, but for the sake of presentation, we used 1 - norm. Furthermore, let $N_{1}=\max _{|\lambda| \leq R} N_{1}(\lambda), N_{2}=\max _{|\lambda| \leq R} N_{2}(\lambda)$, and $N_{R}=$ $\max \left(N_{1}, N_{2}\right)$ in closed contour $\{\lambda \in \mathbb{C}:|\lambda| \leq R\}$; then

$$
\left\|z_{1}(x, \lambda)\right\| \leq 2 M N_{R}(x-c) .
$$

Similarly,

$$
\begin{gathered}
\left\|z_{2}(x, \lambda)\right\| \leq \int_{c}^{x} \mid p_{1}(s)\left(u_{11}-u_{10}\right) \sin \lambda(s-x) \\
\quad-p_{2}(s)\left(u_{21}-u_{20}\right) \cos \lambda(s-x) \mid d s \\
+\int_{c}^{x} \mid p_{1}(s)\left(u_{11}-u_{10}\right) \cos \lambda(s-x) \\
\quad+p_{2}(s)\left(u_{21}-u_{20}\right) \sin \lambda(s-x) \mid d s \\
\leq 2^{2} M^{2} N_{R} \frac{(x-c)^{2}}{2},
\end{gathered}
$$

and so generally,

$$
\left\|z_{n}(x, \lambda)\right\| \leq 2^{n} M^{n} N_{R} \frac{(x-c)^{n}}{n !} .
$$

Now, consider the infinite series

$$
u_{0}(x, \lambda)+\sum_{k=1}^{\infty} z_{k}(x, \lambda)
$$

The $n$th partial sum of this series is $u_{n}(x, \lambda)$; that is,

$$
u_{n}(x, \lambda)=u_{0}(x, \lambda)+\sum_{k=1}^{n} z_{k}(x, \lambda)
$$

Therefore, the sequence $\left\{u_{n}(x, \lambda)\right\}$ converges if and only if series (31) does so. In view of (30), it follows that series (31) is uniformly convergent with respect to $x$ on $(c, b]$ and $\lambda$ in the closed contour $\{\lambda \in \mathbb{C}:|\lambda| \leq R\}$. Let the sum of series (31) be $\Phi_{2}(x, \lambda)=\left(\Phi_{12}(x, \lambda), \Phi_{22}(x, \lambda)\right)^{T}$; that is,

$$
\Phi_{2}(x, \lambda)=u_{0}(x, \lambda)+\sum_{k=1}^{\infty} z_{k}(x, \lambda)
$$

and so, (32) gives

$$
\lim _{n \rightarrow \infty} u_{n}(x, \lambda)=\Phi_{2}(x, \lambda)
$$

Finally, we will show next that the limit function $\Phi_{2}(x, \lambda)$ satisfies (20). For this, we need to find $\Phi_{2}^{\prime}(x, \lambda)$. From (33),

$$
\begin{aligned}
\Phi_{2}^{\prime}(x, \lambda) & =\left(\begin{array}{l}
\Phi_{12}^{\prime}(x, \lambda) \\
\Phi_{22}^{\prime}(x, \lambda)
\end{array}\right) \\
& =\left(\begin{array}{l}
u_{11}^{\prime}(x, \lambda) \\
u_{21}^{\prime}(x, \lambda)
\end{array}\right)+\sum_{k=2}^{\infty}\left(\begin{array}{c}
z_{1 k}^{\prime}(x, \lambda) \\
z_{2 k}^{\prime}(x, \lambda)
\end{array}\right) .
\end{aligned}
$$

For the first term on the right-hand side of (35), if we take $n=1$ in (25), then

$$
\begin{aligned}
& \left(\begin{array}{l}
u_{11} \\
u_{21}
\end{array}\right)=\left(\begin{array}{l}
u_{10} \\
u_{20}
\end{array}\right) \\
& +\int_{c}^{x}\left(\begin{array}{cc}
p_{1}(s) \sin \lambda(s-x) & -p_{2}(s) \cos \lambda(s-x) \\
p_{1}(s) \cos \lambda(s-x) & p_{2}(s) \sin \lambda(s-x)
\end{array}\right) \\
& \times\left(\begin{array}{l}
u_{10} \\
u_{20}
\end{array}\right) d s \\
& \left(\begin{array}{l}
u_{11}^{\prime} \\
u_{21}^{\prime}
\end{array}\right)=\left(\begin{array}{l}
u_{10}^{\prime} \\
u_{20}^{\prime}
\end{array}\right) \\
& +\int_{c}^{x}\left(\begin{array}{cc}
-\lambda p_{1}(s) \cos \lambda(s-x) & -\lambda p_{2}(s) \sin \lambda(s-x) \\
\lambda p_{1}(s) \sin \lambda(s-x) & -\lambda p_{2}(s) \cos \lambda(s-x)
\end{array}\right) \\
& \times\left(\begin{array}{l}
u_{10} \\
u_{20}
\end{array}\right) d s \\
& +\left(\begin{array}{cc}
0 & -p_{2}(x) \\
p_{1}(x) & 0
\end{array}\right)\left(\begin{array}{l}
u_{10} \\
u_{20}
\end{array}\right)
\end{aligned}
$$

now from (25) and the fact that $\left(u_{10}, u_{20}\right)^{T}$ is a solution of the homogeneous system, we have

$$
\left(\begin{array}{l}
u_{11}^{\prime} \\
u_{21}^{\prime}
\end{array}\right)=\left(\begin{array}{cc}
0 & -\lambda \\
\lambda & 0
\end{array}\right)\left(\begin{array}{l}
u_{11} \\
u_{21}
\end{array}\right)+\left(\begin{array}{cc}
0 & -p_{2}(x) \\
p_{1}(x) & 0
\end{array}\right)\left(\begin{array}{l}
u_{10} \\
u_{20}
\end{array}\right) .
$$

For the second term on the right-hand side of (35), it follows from (25) and (26) that

$$
\begin{aligned}
\left(\begin{array}{c}
z_{1 k} \\
z_{2 k}
\end{array}\right)=\int_{c}^{x}\left(\begin{array}{ll}
p_{1}(s) \sin \lambda(s-x) & -p_{2}(s) \cos \lambda(s-x) \\
p_{1}(s) \cos \lambda(s-x) & p_{2}(s) \sin \lambda(s-x)
\end{array}\right) \\
\quad \times\left(\begin{array}{c}
z_{1 k-1} \\
z_{2 k-1}
\end{array}\right) d s
\end{aligned}
$$


Abstract and Applied Analysis

5

and its derivative is

$$
\begin{aligned}
\left(\begin{array}{c}
z_{1 k}^{\prime} \\
z_{2 k}^{\prime}
\end{array}\right)= & \int_{c}^{x}\left(\begin{array}{cc}
-\lambda p_{1}(s) \cos \lambda(s-x) & -\lambda p_{2}(s) \sin \lambda(s-x) \\
\lambda p_{1}(s) \sin \lambda(s-x) & -\lambda p_{2}(s) \cos \lambda(s-x)
\end{array}\right) \\
& \times\left(\begin{array}{c}
z_{1 k-1} \\
z_{2 k-1}
\end{array}\right) d s \\
& +\left(\begin{array}{cc}
0 & -p_{2}(x) \\
p_{1}(x) & 0
\end{array}\right)\left(\begin{array}{c}
z_{1 k-1} \\
z_{2 k-1}
\end{array}\right) .
\end{aligned}
$$

In this equation

$$
\begin{gathered}
\int_{c}^{x}\left(\begin{array}{cc}
-\lambda p_{1}(s) \cos \lambda(s-x) & -\lambda p_{2}(s) \sin \lambda(s-x) \\
\lambda p_{1}(s) \sin \lambda(s-x) & -\lambda p_{2}(s) \cos \lambda(s-x)
\end{array}\right) \\
\times\left(\begin{array}{c}
z_{1 k-1} \\
z_{2 k-1}
\end{array}\right) d s=\left(\begin{array}{cc}
0 & -\lambda \\
\lambda & 0
\end{array}\right)\left(\begin{array}{l}
z_{1 k} \\
z_{2 k}
\end{array}\right) .
\end{gathered}
$$

By using (39) and (40), the second term on the right-hand side of (35) becomes

$$
\begin{aligned}
\sum_{n=2}^{\infty}\left(\begin{array}{c}
z_{1 k}^{\prime}(x, \lambda) \\
z_{2 k}^{\prime}(x, \lambda)
\end{array}\right)= & \left(\begin{array}{cc}
0 & -\lambda \\
\lambda & 0
\end{array}\right) \sum_{k=2}^{\infty}\left(\begin{array}{l}
z_{1 k} \\
z_{2 k}
\end{array}\right) \\
& +\left(\begin{array}{cc}
0 & -p_{2}(x) \\
p_{1}(x) & 0
\end{array}\right) \sum_{k=2}^{\infty}\left(\begin{array}{l}
z_{1 k-1} \\
z_{2 k-1}
\end{array}\right) \\
= & \left(\begin{array}{cc}
0 & -\lambda \\
\lambda & 0
\end{array}\right)\left[\sum_{k=1}^{\infty}\left(\begin{array}{l}
z_{1 k} \\
z_{2 k}
\end{array}\right)-\left(\begin{array}{l}
z_{11} \\
z_{21}
\end{array}\right)\right] \\
& +\left(\begin{array}{cc}
0 & -p_{2}(x) \\
p_{1}(x) & 0
\end{array}\right) \sum_{k=1}^{\infty}\left(\begin{array}{l}
z_{1 k} \\
z_{2 k}
\end{array}\right) .
\end{aligned}
$$

Substituting (37) and (41) into (35) gives

$$
\begin{aligned}
& \left(\begin{array}{l}
\Phi_{12}^{\prime}(x, \lambda) \\
\Phi_{22}^{\prime}(x, \lambda)
\end{array}\right)=\left(\begin{array}{cc}
0 & -\lambda \\
\lambda & 0
\end{array}\right)\left[\left(\begin{array}{l}
u_{11} \\
u_{21}
\end{array}\right)-\left(\begin{array}{l}
z_{11} \\
z_{21}
\end{array}\right)\right] \\
& +\left(\begin{array}{cc}
0 & -p_{2}(x) \\
p_{1}(x) & 0
\end{array}\right)\left(\begin{array}{l}
u_{10} \\
u_{20}
\end{array}\right) \\
& +\left(\begin{array}{cc}
0 & -\lambda \\
\lambda & 0
\end{array}\right) \sum_{k=1}^{\infty}\left(\begin{array}{l}
z_{1 k} \\
z_{2 k}
\end{array}\right) \\
& +\left(\begin{array}{cc}
0 & -p_{2}(x) \\
p_{1}(x) & 0
\end{array}\right) \sum_{k=1}^{\infty}\left(\begin{array}{l}
z_{1 k} \\
z_{2 k}
\end{array}\right) \\
& =\left(\begin{array}{cc}
0 & -\lambda \\
\lambda & 0
\end{array}\right)\left(\begin{array}{l}
u_{10} \\
u_{20}
\end{array}\right)+\left(\begin{array}{cc}
0 & -p_{2}(x) \\
p_{1}(x) & 0
\end{array}\right)\left(\begin{array}{l}
u_{10} \\
u_{20}
\end{array}\right) \\
& +\left(\begin{array}{cc}
0 & -\lambda-p_{2}(x) \\
\lambda+p_{1}(x) & 0
\end{array}\right) \sum_{k=1}^{\infty}\left(\begin{array}{l}
z_{1 k} \\
z_{2 k}
\end{array}\right) \\
& =\left(\begin{array}{cc}
0 & -\lambda-p_{2}(x) \\
\lambda+p_{1}(x) & 0
\end{array}\right)
\end{aligned}
$$

$$
\begin{aligned}
& \times\left[\left(\begin{array}{l}
u_{10} \\
u_{20}
\end{array}\right)+\sum_{k=1}^{\infty}\left(\begin{array}{l}
z_{1 k} \\
z_{2 k}
\end{array}\right)\right] \\
= & \left(\begin{array}{cc}
0 & -\lambda-p_{2}(x) \\
\lambda+p_{1}(x) & 0
\end{array}\right)\left(\begin{array}{l}
\Phi_{12}(x, \lambda) \\
\Phi_{22}(x, \lambda)
\end{array}\right)
\end{aligned}
$$

so that $\Phi_{2}(x, \lambda)$ satisfies $(20)$ on $(c, b]$. It also clearly satisfies the boundary conditions (21). As a result, the vector-valued function $\Phi(x, \lambda)$ defined by

$$
\Phi(x, \lambda)= \begin{cases}\Phi_{1}^{T}(x, \lambda)=\left(\Phi_{11}, \Phi_{21}\right), & x \in[a, c), \\ \Phi_{2}^{T}(x, \lambda)=\left(\Phi_{12}, \Phi_{22}\right), & x \in(c, b]\end{cases}
$$

satisfies the Dirac system (1), (4), and (6).

Theorem 6. For any $\lambda \in \mathbb{C}$, the Dirac system

$$
\begin{aligned}
& u_{2}^{\prime}(x)-p_{1}(x) u_{1}(x)=\lambda u_{1}(x), \\
& u_{1}^{\prime}(x)+p_{2}(x) u_{2}(x)=-\lambda u_{2}(x)
\end{aligned}
$$

has a solution

$$
\Psi(x, \lambda)= \begin{cases}\Psi_{1}^{T}(x, \lambda)=\left(\Psi_{11}, \Psi_{21}\right), & x \in[a, c), \\ \Psi_{2}^{T}(x, \lambda)=\left(\Psi_{12}, \Psi_{22}\right), & x \in(c, b]\end{cases}
$$

on $[a, c) \cup(c, b]$ satisfying the boundary condition (5) and transmission condition (6). For each $x \in[a, c) \cup(c, b], \Psi(x, \lambda)$ is a vector-valued entire function of $\lambda$.

Proof. The proof of this theorem is similar to that of Theorem 5 and hence is omitted.

4. The Eigenvalues of the Problem

We know from [11, page 194] that the Wronskians $W\left(\Phi_{i}, \Psi_{i}\right)$, $(i=1,2)$ do not depend on $x \in[a, c) \cup(c, b]$. They depend only on $\lambda$, and let $W\left(\Phi_{i}(x, \lambda), \Psi_{i}(x, \lambda)\right)=: \omega_{i}(\lambda)(i=1,2)$. However, it follows from (6) that

$$
\begin{aligned}
\omega_{1}(\lambda)= & W\left(\Phi_{1}, \Psi_{1}\right)=\left|\begin{array}{ll}
\Phi_{11}(x, \lambda) & \Phi_{21}(x, \lambda) \\
\Psi_{11}(x, \lambda) & \Psi_{21}(x, \lambda)
\end{array}\right| \\
= & \Phi_{11}(c-0, \lambda) \Psi_{21}(c-0, \lambda) \\
& -\Phi_{21}(c-0, \lambda) \Psi_{11}(c-0, \lambda) \\
= & \gamma^{-1} \Phi_{12}(c+0, \lambda) \gamma \Psi_{22}(c+0, \lambda) \\
& -\gamma \Phi_{22}(c+0, \lambda) \gamma^{-1} \Psi_{12}(c+0, \lambda) \\
= & \left|\begin{array}{ll}
\Phi_{12}(x, \lambda) & \Phi_{22}(x, \lambda) \\
\Psi_{12}(x, \lambda) & \Psi_{22}(x, \lambda)
\end{array}\right|=W\left(\Phi_{2}, \Psi_{2}\right)=\omega_{2}(\lambda) .
\end{aligned}
$$


Hence, we get

$$
\omega_{1}(\lambda)=\omega_{2}(\lambda):=\omega(\lambda) .
$$

Here we defined a function $\omega(\lambda)$.

Let the solutions $\Phi(x, \lambda)$ and $\Psi(x, \lambda)$ of (1)-(6) be defined by the initial conditions for some $\alpha, \beta \in[0, \pi)$

$$
\begin{aligned}
\Phi_{11}(a, \lambda)=\cos \alpha, & \Phi_{21}(a, \lambda)=\sin \alpha \\
\Psi_{12}(b, \lambda)=a_{1}+\lambda \cos \beta, & \Psi_{22}(b, \lambda)=b_{1}+\lambda \sin \beta .
\end{aligned}
$$

Therefore, any solution of (1)-(6) may be represented as

$$
\begin{aligned}
& u(x, \lambda) \\
& \quad= \begin{cases}u_{1}^{T}(x, \lambda)=\left(c_{1} \Phi_{11}+c_{2} \Psi_{11}, c_{1} \Phi_{21}+c_{2} \Psi_{21}\right), & x \in[a, c) \\
u_{2}^{T}(x, \lambda)=\left(c_{3} \Phi_{12}+c_{4} \Psi_{12}, c_{3} \Phi_{22}+c_{4} \Psi_{22}\right), & x \in(c, b] .\end{cases}
\end{aligned}
$$

Applying conditions (4), (5), and (6) to solution (49) and considering the initial values (48), we obtain the following coefficients matrix of linear system equations of the variables $c_{1}, c_{2}, c_{3}, c_{4}$ :

$$
\left[\begin{array}{cccc}
0 & \omega_{1}(\lambda) & 0 & 0 \\
0 & 0 & \omega_{2}(\lambda) & 0 \\
\Phi_{11}(c-0, \lambda) & \Psi_{11}(c-0, \lambda) & -\gamma \Phi_{12}(c+0, \lambda) & -\gamma \Psi_{12}(c+0, \lambda) \\
\Phi_{21}(c-0, \lambda) & \Psi_{21}(c-0, \lambda) & -\gamma^{-1} \Phi_{22}(c+0, \lambda) & -\gamma^{-1} \Psi_{22}(c+0, \lambda)
\end{array}\right]
$$

and let us denote the determinant of this matrix by $W(\lambda)$; then for every $\lambda \in \mathbb{C}$,

$$
\begin{aligned}
W(\lambda) & =-\omega_{1}(\lambda) \omega_{2}(\lambda)\left|\begin{array}{cc}
\Phi_{11}(c-0, \lambda) & \Psi_{11}(c-0, \lambda) \\
\Phi_{21}(c-0, \lambda) & \Psi_{21}(c-0, \lambda)
\end{array}\right| \\
& =-\omega_{1}^{2}(\lambda) \omega_{2}(\lambda)=-\omega^{3}(\lambda) .
\end{aligned}
$$

Theorem 7. The eigenvalues of the problem (1)-(6) are the zeros of the function $\omega(\lambda)$.

Proof. Let $\omega\left(\lambda_{n}\right)=0$ for any $\lambda=\lambda_{n}$. Then, it follows from (51) that the Wronskian of $\Phi_{2}\left(x, \lambda_{n}\right)$ and $\Psi_{2}\left(x, \lambda_{n}\right)$ is zero, so that $\Psi_{2}\left(x, \lambda_{n}\right)$ is a constant multiple of $\Phi_{2}\left(x, \lambda_{n}\right)$, say

$$
\Psi_{2}\left(x, \lambda_{n}\right)=k \Phi_{2}\left(x, \lambda_{n}\right), \quad x \in(c, b] .
$$

It follows that $\Psi\left(x, \lambda_{n}\right)$ also fulfils the boundary condition (5) and, therefore, is a vector-valued eigenfunction of the problem (1)-(6) for eigenvalue $\lambda_{n}$.

Conversely, let $u_{n}\left(x, \lambda_{n}\right)$ be a vector-valued eigenfunction corresponding to eigenvalue $\lambda_{n}$, but $\omega\left(\lambda_{n}\right) \neq 0$. Then, from (51), at least one of the pair of the functions $\left(\Phi_{1}^{T}, \Phi_{2}^{T}\right)$ and $\left(\Psi_{1}^{T}, \Psi_{2}^{T}\right)$ would be linearly independent. Therefore, $u_{n}\left(x, \lambda_{n}\right)$ can be expressed as

$$
u_{n}\left(x, \lambda_{n}\right)= \begin{cases}C_{1} \Phi_{1}^{T}\left(x, \lambda_{n}\right)+C_{2} \Psi_{1}^{T}\left(x, \lambda_{n}\right), & x \in[a, c), \\ D_{1} \Phi_{2}^{T}\left(x, \lambda_{n}\right)+D_{2} \Psi_{2}^{T}\left(x, \lambda_{n}\right), & x \in(c, b],\end{cases}
$$

where at least one of the constants $C_{1}, C_{2}, D_{1}, D_{2}$ is not zero. Since $u_{n}\left(x, \lambda_{n}\right)$ is a vector-valued eigenfunction corresponding to eigenvalue $\lambda_{n}$ by substitution in conditions (4)-(6), we obtain a system of linear, homogeneous equations and the determinant of this system is zero. This means that $W\left(\lambda_{n}\right)=$ 0 , and from (51), $\omega\left(\lambda_{n}\right)=0$ which yields a contradiction to the assumption that $\omega\left(\lambda_{n}\right) \neq 0$. This completes the proof.

Since $\omega(\lambda)$ is an entire function of $\lambda$ and the eigenvalues of the problem (1)-(6) consist of the zeros of $\omega(\lambda)$, we have the next theorem.

Theorem 8. The Dirac system (1)-(6) has at most denumerably many eigenvalues, and these eigenvalues have no finite limit point.

\section{References}

[1] J. Walter, "Regular eigenvalue problems with eigenvalue parameter in the boundary condition," Mathematische Zeitschrift, vol. 133, pp. 301-312, 1973.

[2] C. T. Fulton, "Two-point boundary value problems with eigenvalue parameter contained in the boundary conditions," Proceedings of the Royal Society of Edinburgh A, vol. 77, no. 3-4, pp. 293-308, 1977.

[3] C. T. Fulton, "Singular eigenvalue problems with eigenvalue parameter contained in the boundary conditions," Proceedings of the Royal Society of Edinburgh A, vol. 87, no. 1-2, pp. 1-34, 1980.

[4] E. C. Titchmarsh, Eigenfunction Expansions Associated with Second-Order Differential Equations, Clarendon Press, Oxford, UK, 1962.

[5] O. Sh. Mukhtarov and E. Tunç, "Eigenvalue problems for Sturm-Liouville equations with transmission conditions," Israel Journal of Mathematics, vol. 144, pp. 367-380, 2004.

[6] Z. Akdoğan, M. Demirci, and O. Sh. Mukhtarov, "Discontinuous Sturm-Liouville problems with eigenparameter-dependent boundary and transmissions conditions," Acta Applicandae Mathematicae, vol. 86, no. 3, pp. 329-344, 2005.

[7] M. Kadakal and O. Sh. Mukhtarov, "Sturm-Liouville problems with discontinuities at two points," Computers \& Mathematics with Applications, vol. 54, no. 11-12, pp. 1367-1379, 2007.

[8] N. B. Kerimov, "On a boundary value problem for the Dirac system with a spectral parameter in the boundary conditions," Differential Equations, vol. 38, no. 2, pp. 164-174, 2002.

[9] B. Keskin and A. S. Özkan, "Inverse spectral problems for Dirac operator with eigenvalue dependent boundary and jump conditions," Acta Mathematica Hungarica, vol. 130, no. 4, pp. 309-320, 2011.

[10] M. Rama Mohana Rao, Ordinary Differential Equations-Theory and Applications, Edward Arnold, London, UK, 1980.

[11] B. M. Levitan and I. S. Sargsjan, Sturm-Liouville and Dirac perators, vol. 59, Kluwer Academic Publishers Group, Boston, Mass, USA, 1991. 


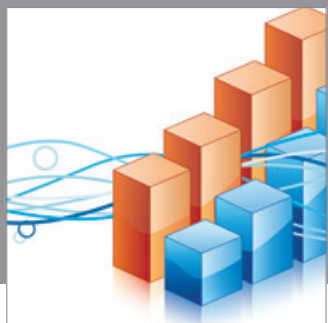

Advances in

Operations Research

mansans

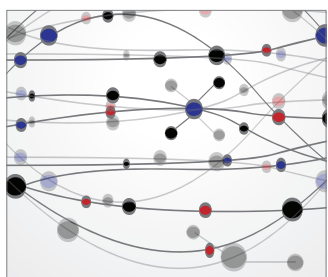

The Scientific World Journal
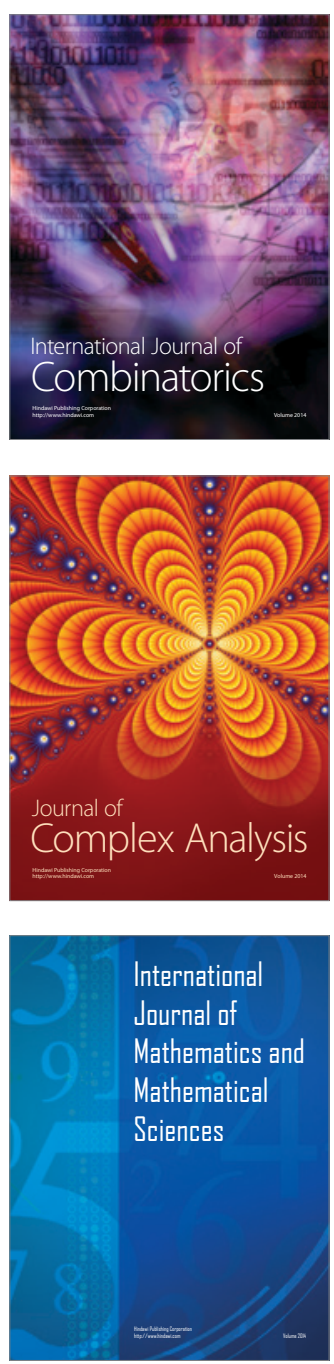
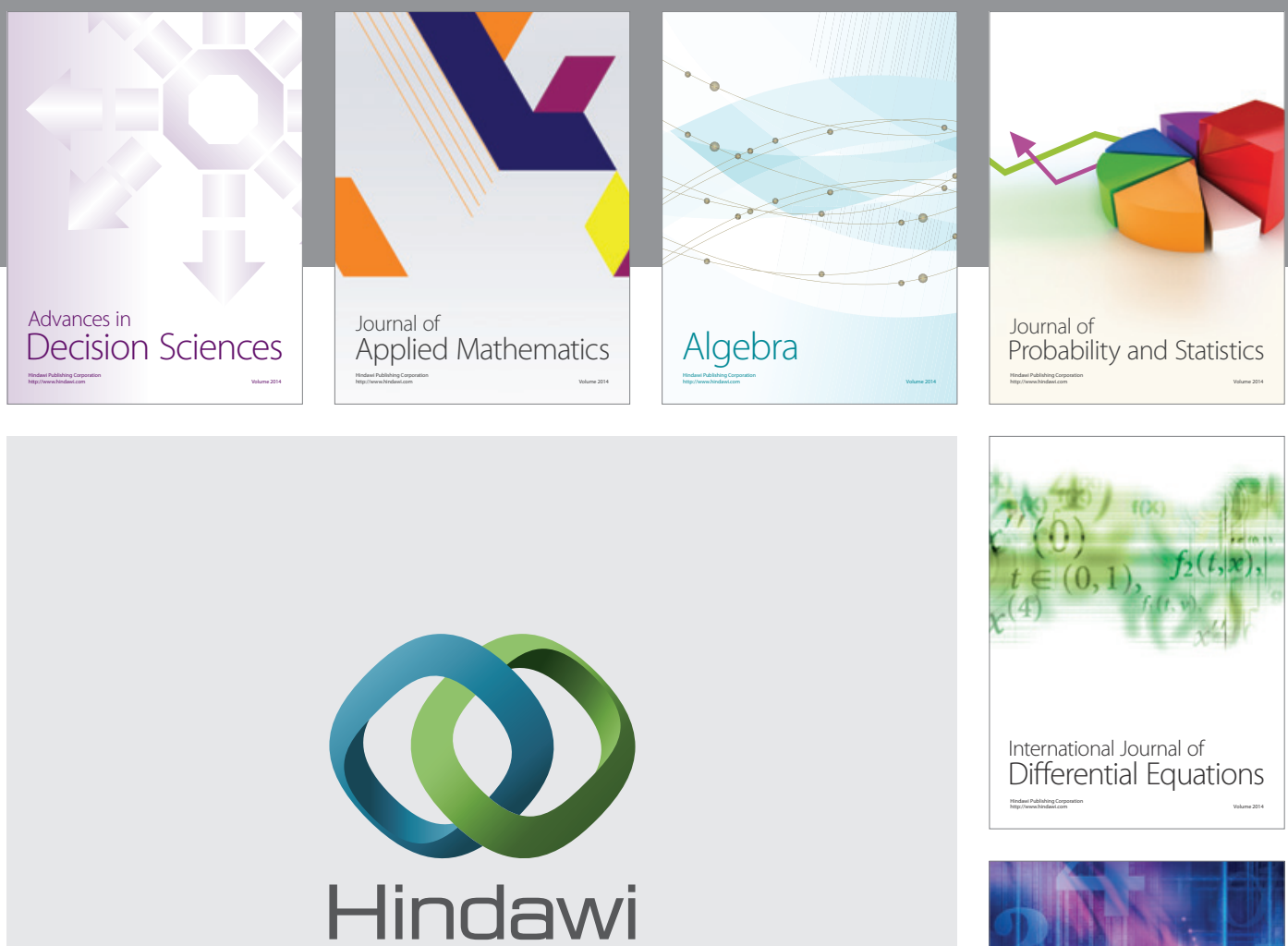

Submit your manuscripts at http://www.hindawi.com
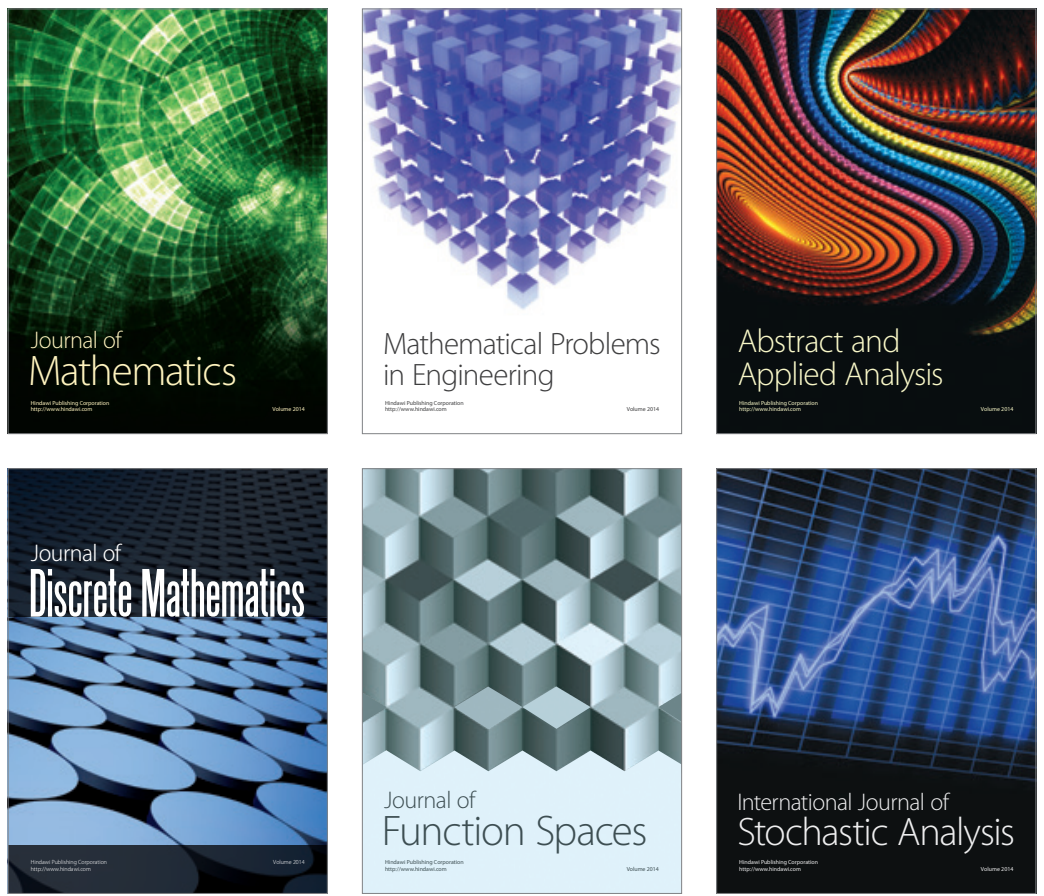

Journal of

Function Spaces

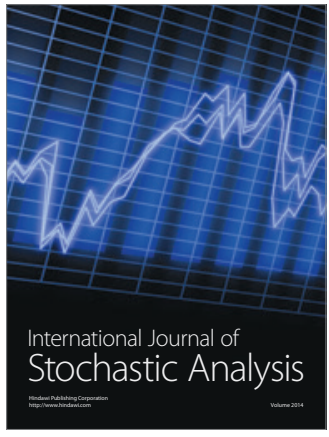

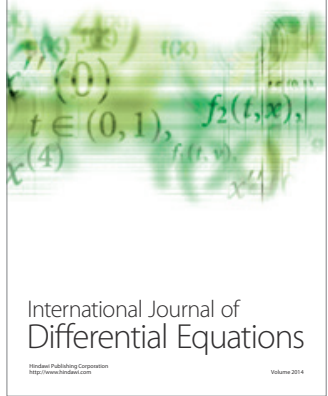
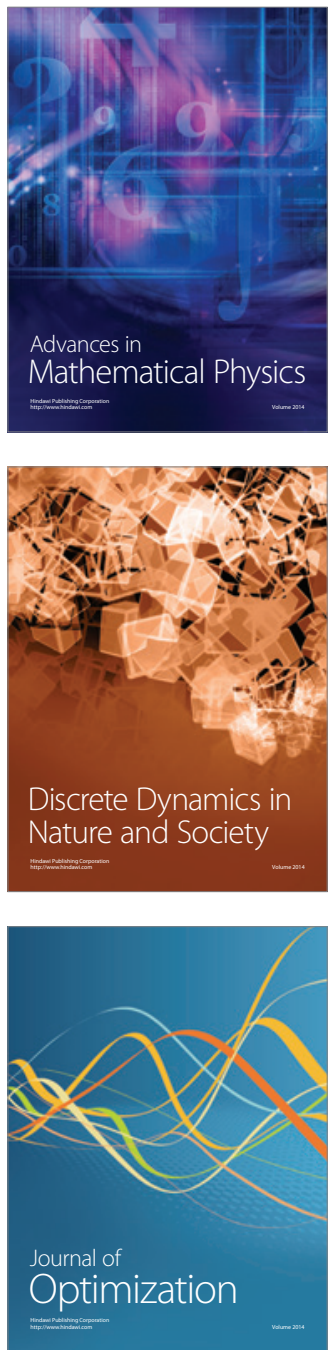